\title{
POLARIZATION OF DUSTY "QUASIATOMS" IN LOW-DENSITY PLASMA
}

\author{
G.I. SUKHININ, ${ }^{1,2}$ A.V. FEDOSEEV ${ }^{1}$ \\ ${ }^{\mathbf{1}}$ S. Kutateladze Institute of Thermophysics SB RAS \\ (1, Lavrentyev Ave., Novosibirsk 630090, Russia; e-mail: sukhinin@itp.nsc.ru) \\ ${ }^{2}$ Novosibirsk State University \\ (2, Pirogova Str., Novosibirsk 630090, Russia)
}

PACS 52.27

(C) 2011

A model of the polarization of dust particles with trapped ions in an external electric field is presented. It is based on the selfconsistent solution of the integral balance equation for trapped ion density and Poisson equation for the electric potential. It is shown that, in a low collisional regime, trapped ions form a spread shell with a maximum located at some distance from the charged dust particle proportional to the ion Debye length. The estimated polarizability of dusty "quasiatoms" is very high and depends on the strength of the external electric field due to the field ionization of trapped ions. With increase in the electric field, the dusty "quasiatom" loses their trapped ions from far orbits due to the field ionization. The radius of the dusty "quasiatom" ionic shell becomes smaller, and the coefficient of polarizability decreases.

\section{Introduction}

Dusty or complex plasma is the ionized gas of neutrals, electrons, ions, and negatively charged micronsized particles. Dusty grains can be found in space (planet rings, cometary tails, and interstellar molecular clouds) or in different technological installations (plasma chemical and thermonuclear reactors). Under laboratory conditions, dusty plasma is investigated in RF plasma and in DC glow discharges [1-3]. Many intriguing phenomena are observed in complex plasmas: the formation of ordered (crystal-like or liquid-like) structures, phase transitions, propagation of low-frequency waves, vortices, and formation of voids.

Electric field plays a paramount role in dusty plasma under laboratory conditions. The value of a reduced electric field, $E / N_{g}$, determines the electron energy distribution function (EEDF) and the ion velocity distribution function in low-density plasma, which determine the charge of dust particles, $Z_{d}$. In particular, the electric fields in discharge plasma are responsible for the difference between the mean energies of electrons and ions $\left(T_{e}\right.$ is much higher than $T_{i}$ ). Moreover, the EEDF in lowdensity discharges is usually non-equilibrium and cannot be described as the Maxwellian distribution.
In plasma, an external electric field provides electron and ion flows with drift velocities (far from a dust particle) proportional to the strength of the electric field. The ion flow in an external electric field leads to the breakdown of spherical symmetry in the space around dust particles. For the simplest case of a single dust particle, the spherical symmetry changes to cylindrical symmetry. The ion flow in the electric field leads to shadowing effects and the formation of a wake structure behind the dust particle, which may be responsible for the structure formation in dusty plasma [4-6].

Another important influence of an external electric field on a dust particle is the formation of a dipole moment in the system of a negatively charged particletrapped ion cloud. It is known that ions can lose energy in rare collisions with atoms and become trapped in finite orbits by the electric field of a charged particle [7-9]. The problem of trapped ions in a low-collisional case was studied by Zobnin [10] and by M. Lampe [1113]. It was shown that the total number $Z_{t r}$ of trapped ions can be comparable to the proper charge number of a dust particle, $Z_{t r} \approx 0.5 Z_{d}$. However, it should be mentioned that, in collisional regimes in plasma, an additional flux of trapped ions after their charge exchange collisions with atoms appeared to reduce the charge of a dust grain in comparison with that predicted in the OML theory $[14,15]$.

In this paper, we estimate the polarizability of "dust grain + cloud of trapped ions" or a "dusty quasiatom" in an external electric field in almost collisionless plasma taking the radial distribution of trapped ions into account. For determining the trapped ion radial distribution, the numerical model presented in [16] and corrected in [17] is used. The model is based on the balance equation describing the formation and the destruction of trapped ions in charge exchange collisions with neutral atoms in low-density plasma and on the Poisson equation for the electric potential. 

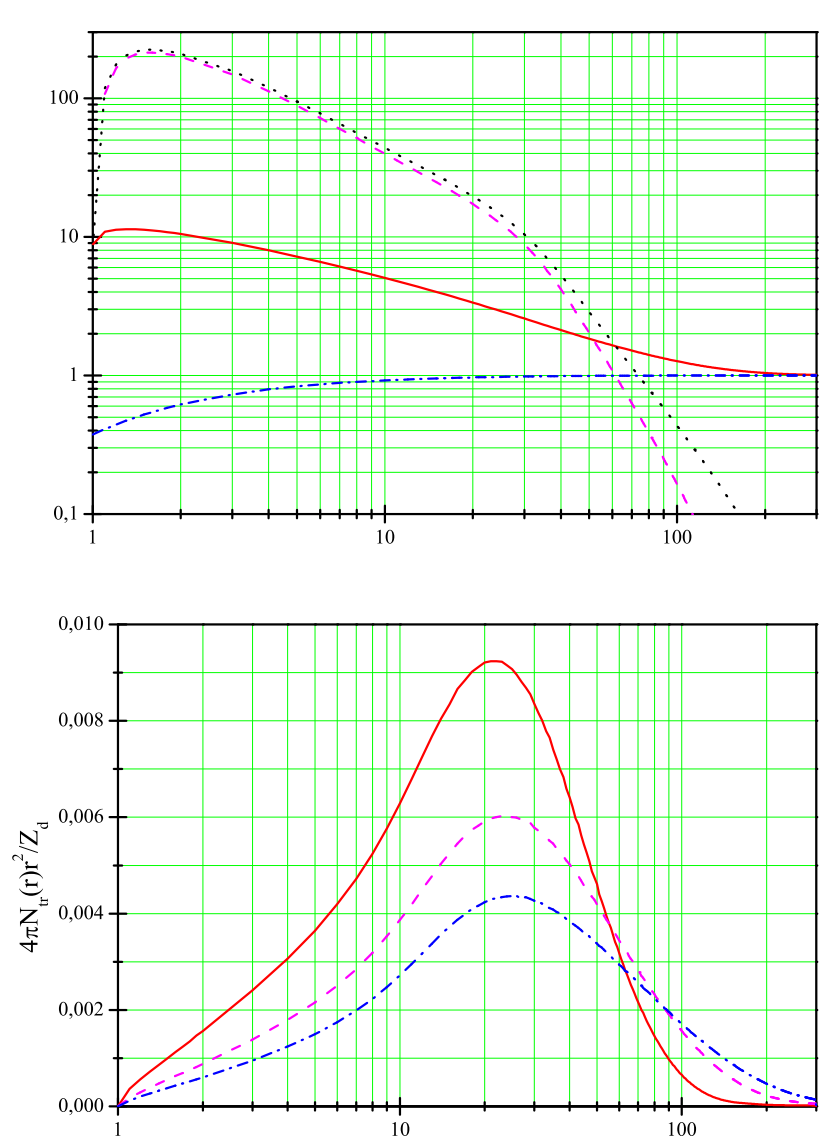

Fig. 1. a) Radial distributions of charged particles densities: free ions $N_{\text {if }}(r)$ (solid line), trapped ions $N_{\operatorname{tr}}(r)$ (dashed line), electrons $N_{e}(r)$ (dash-dotted line) and volume charge $\Delta N(r)$ (dotted line). $\lambda_{i}=66 r_{0}$. b) Radial distribution of the function $4 \pi N_{\operatorname{tr}}(r) r^{2}$ for different Debye lengths $\lambda_{i}=33 r_{0}$ (solid line), 66ro (dashed line), and $99 r_{0}$ (dash-dotted line)

\section{Polarizability of a Dusty Quasiatom}

In paper [17], it was shown that, in a low collisional regime, trapped ions form a spread shell with a maximum located at some distance from the charged dust particle proportional to the ion Debye length, $R_{t r} \approx$ $(0.25-0.5) \lambda_{i}$. It means that dusty "quasiatom" is formed $[12,17]$, which can be polarized in an external electric field, leading to van der Waals-type attractive forces between grains. In Fig. 1, $a$, the example of radial distributions of charged particles densities are presented for different ion Debye lengths and $Z_{d}=5000$. In Fig. 1,b, the radial distributions of the function $4 \pi N_{t r}(r) r^{2}$ are presented for the same Debye lengths, where $N_{t r}(r)$ is the trapped ion density.
To estimate the polarizability of a dusty quasiatom, we assume for simplicity that the strength of an external electric field is not large and does not disturb very much the spherical distribution of trapped ions in a vicinity of the dust particle.

An individual trapped ion moves in the well of the effective potential $e \varphi_{\text {eff }}(r)=e \varphi(r)+J^{2} / 2 M r^{2}$ with minimum at $r_{m}\left(\varphi_{\text {eff }}^{\prime}\left(r_{m}\right)=0\right)$ between turning points $r_{\min }$ and $r_{\max }$. Here, $\varphi(r)$ is a self-consistent potential around a dust particle, $J$ and $M$ are the angular momentum and the mass of this ion, $r_{0}$ is the radius of a micron-sized dust particle. It is known $[12,17]$ that, without external electric field, the radial distribution of the self-consistent potential around a dust particle has Debye-Hückel-like form. Below for the qualitative consideration of the dusty "quasiatom" polarization, we will use the Debye-Hückel potential (DH-potential) $\varphi(r)=-e Z_{d} \exp \left(-\left(r-r_{0}\right) / \lambda_{i}\right) / r$.

Ion can be trapped only for $J_{\min }^{2}<J^{2}<J_{\max }^{2}$, and the potential well can be formed only in the range $r_{0} \leq r_{m} \leq$ $R_{m}$ (for Debye-Hückel potential, $R_{m}=\lambda_{i}\left(1+5^{1 / 2}\right) / 2 \approx$ $\left.1.618 \lambda_{i}\right)$. There is no potential well for ion trajectories with angular momentum $J^{2} \geq J_{\max }^{2} \approx 0.84 M_{i} \lambda_{i}^{2}$, and such an ion cannot be trapped by a charged particle.

In Fig. 2, four effective potentials for different values of angular momenta are presented. Two curves corresponds to $r_{m}=0.3 \lambda_{i}$ and $0.5 \lambda_{i}\left(J^{2}<J_{\max }^{2}\right)$, the solid line presents the critical effective potential with $J^{2}=J_{\max }^{2}$ $\left(r_{m}=R_{m}\right)$, and the last curve (symbols) presents the effective potential in the case where the ion cannot be trapped, $J^{2}=1.5 J_{\max }^{2}$. It is seen that ions belonging to some $r_{m}<R_{m}$ can have the total energy in the interval from minimum value, $W_{\min }\left(r_{m}\right)=e \varphi_{\text {eff }}\left(r_{m}\right)$, to some positive small value at the centrifugal barrier, $W_{\max }\left(r_{m}\right)$. The maximum value of barrier energy (for DH-potential) is equal to $W_{\max }\left(R_{m}\right)=0,0757 e^{2} Z_{d} / 2 \lambda_{i}$ that usually has the order of $0.1 T_{e}\left(r_{0} / \lambda_{i}\right) \leq T_{i}$.

The effective potential in a vicinity of $r \approx r_{m}$ can be approximated as a quadratic form

$\varphi_{\mathrm{eff}}(r)=\varphi_{\mathrm{eff}}\left(r_{m}\right)+\frac{1}{2} \varphi_{\mathrm{eff}}^{\prime \prime}\left(r_{m}\right)\left(r-r_{m}\right)^{2}$

with $\varphi_{\text {eff }}^{\prime}(r)=0$ and $\varphi_{\text {eff }}^{\prime \prime}\left(r_{m}\right)>0$. For the DH-potential, $\varphi_{\text {eff }}^{\prime \prime}\left(r_{m}\right)$ can be presented in the form

$\varphi_{\mathrm{eff}}\left(r_{m}\right)=\beta\left(r_{m} / \lambda_{i}\right) \widetilde{Z}_{d} e / r_{m}^{3}$

where the function $\beta\left(r_{m} / \lambda_{i}\right)$ has the order of 1 for $r_{m} / \lambda_{i}<1$, and $\beta\left(r_{m} / \lambda_{i}\right) \rightarrow 0$ for $r_{m} \rightarrow R_{m}$. For the DH-potential, the function $\beta(x)$ is equal to $\beta(x)=$ $\left(1+x-x^{2}\right) \exp (-x)$. 


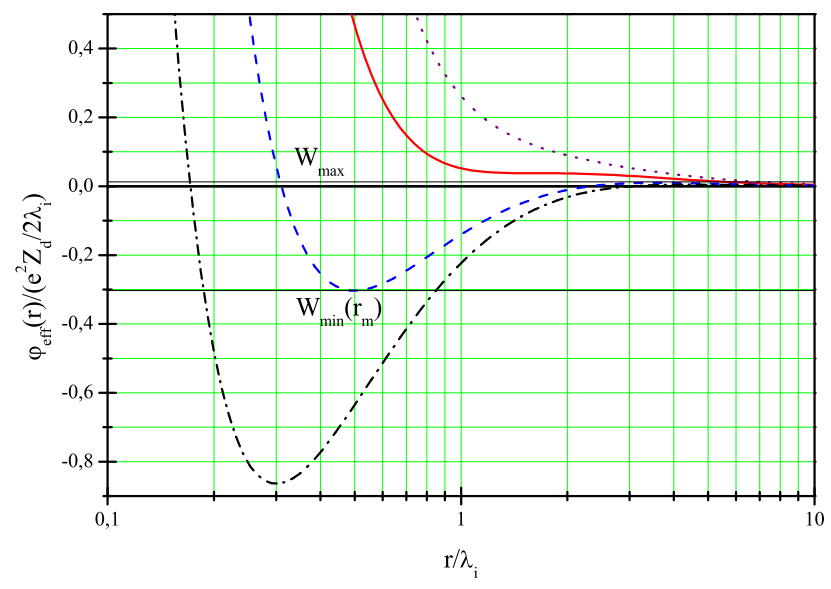

Fig. 2. Effective potentials $\varphi_{\text {eff }}(r)$ for $r_{m}=0.3 \lambda_{i}$ (dash-dotted line), $0.5 \lambda_{i}$ (dashed line), and $R_{m}=1.618 \lambda_{i}$ (solid line). Horizontal lines present the minimal and maximal total energies of an ion with the angular momentum corresponding to $r_{m}=0.5 \lambda_{i}$

Under the action of an external electric field $E$, a trapped ion is displaced from its stationary orbit. The elastic restoring force acting on a trapped ion in this potential is equal to $F_{r}=-e \varphi_{\text {eff }}^{\prime \prime}\left(r_{m}\right)\left(r-r_{m}\right)=-e E$. Such an ion has a dipole moment equal to

$\mathbf{p}\left(r_{m}\right)=e\left(\mathbf{r}-\mathbf{r}_{m}\right)=e \mathbf{E} / \varphi_{\mathrm{eff}}^{\prime \prime}\left(r_{m}\right)$

Formally, ions with parameters of orbits in a vicinity of $R_{m}$ should have, according to , a very large polarizability. However, for $r_{m} \rightarrow R_{m}$, the effective potential well is very shallow. For example, for $r_{m}=\lambda_{i}$, the potential well depth is equal to $0.027 e^{2} \widetilde{Z}_{d} / \lambda_{i} \sim 0.1 T_{e} r_{0} / \lambda_{i}$. Usually, for $\lambda_{i} / r_{0}>10$, this value is smaller than the gas and ion temperatures. Ions cannot be trapped into such a well and will be ionized either due to collisions with neutrals or due to gaining the kinetic energy moving along their orbits in the electric field.

After the averaging over all trapped ions $Z_{t r}$, which have the radial distribution $N_{t r}\left(r_{m}\right)$, the dipole moment of a "quasiatom" can be estimated as

$\mathbf{P}_{\mathrm{tr}}=4 \pi e \mathbf{E} \int_{r_{0}}^{R_{E}} d r_{m} r_{m}^{2} \frac{N_{\mathrm{tr}}\left(r_{m}\right)}{\varphi_{\mathrm{eff}}^{\prime \prime}\left(r_{m}\right)}=\alpha_{d}(E) \mathbf{E}$.

The upper limit in the integral, $R_{E}$, can be chosen from the following consideration. With increase in the external electric field, $E$, trapped ions on its finite orbit can gain the energy $\Delta U_{E}=\delta^{2} e E r_{m}$, where $\delta \leq 1$ depends on the exact solution of the ion dynamics in the external field (in this paper, $\delta=0.5$ ).

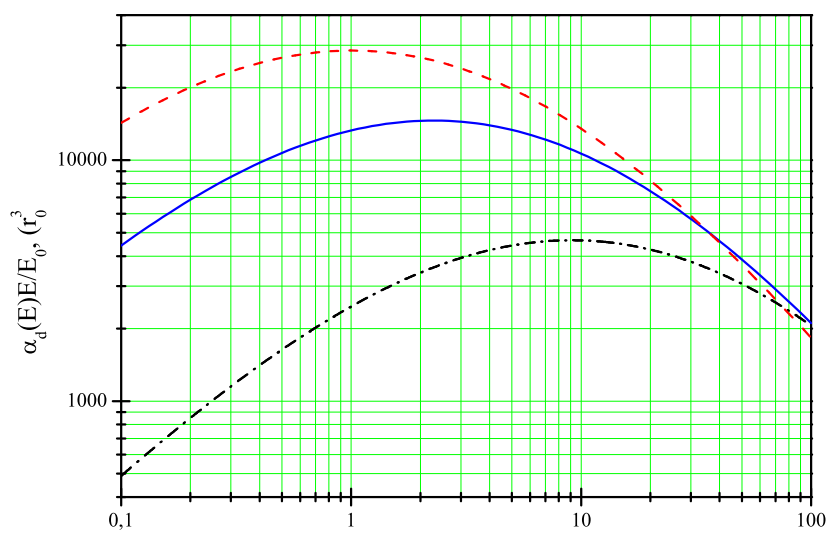

Fig. 3. Dependence of the dipole moment of a dusty "quasiatom" on the strength of an external electric field for $\lambda_{i}=33 r_{0}$ (dashdotted line), $\lambda_{i}=66 r_{0}$ (solid line), and $\lambda_{i}=99 r_{0}$ (dashed line), $\delta=0.5$

If the gain $\Delta U_{E}$ is higher than the depth of a potential well, the trapped ion will be transferred to an infinite orbit. The boundary value of radius $R_{E}$ can be approximated as $\left.R_{E} \approx \lambda_{i} /\left(1+\delta\left(E / E_{0}\right)^{1 / 2} \lambda_{i} / 66 r_{0}\right)\right)$, $\left(E_{0}=e Z_{d} / 2\left(66 r_{0}\right)^{2},\left(E_{0} \approx 8.3 \mathrm{~V} / \mathrm{cm}\right.\right.$ for $Z_{d}=5000$, $\left.r_{0}=10^{-4} \mathrm{~cm}, \lambda_{i}=66 r_{0}\right)$. Using the obtained results for $N_{\mathrm{tr}}(r)$, we can approximate the coefficient of polarizability for different $\lambda_{i}$ :

$\alpha_{d}(E) \approx A\left(r_{0} / \lambda_{i}\right)\left(R_{E} / r_{0}\right)^{\gamma} r_{0}^{3} \gg r_{0}^{3}$,

where $A \approx 0.020 \pm 0.003, \gamma \approx 4.65 \pm 0.15$. The obtained estimation shows that the coefficient of polarizability depends on the external electric field via the field dependence of $R_{E}$. The normalized dependence of the dipole moment $\mathbf{P}_{\mathrm{tr}}(E)=\alpha_{d}(E) \mathbf{E}$ on the electric field is presented in Fig. 3. It is seen that the dipole moment has a maximum at $E_{\max } \approx E_{0}\left(50 r_{0} / \lambda_{i} \delta\right)^{2}$. The meaning of the nonlinear dependence (5) is the following: as the electric field increases, the dusty "quasiatom" loses their trapped ions from far orbits due to the field ionization. The radius of the dusty "quasiatom" ionic shell becomes smaller and the coefficient of polarizability decreases.

The dipole-dipole attractive force is comparable to the shadowing force that is connected with the reciprocal interception of ions by the neighboring dust particles and is much higher than the dipole-dipole interaction of polarized dielectric grains induced by the non-uniform charging [18]. The attractive force for two identical parallel dipoles $\mathbf{P}$ separated by distance $R$ is equal to $F_{d d}=6 P^{2} / R^{4}$. According to [18], the shadowing force is $F_{s h}=3 / 8\left(r_{0} / \lambda_{i}\right)^{2}\left(e Z_{d} / R\right)^{2}$. The ratio of the dipole- 
dipole and shadowing forces can be presented in the form

$F_{d d} / F_{\text {sh }} \approx 21\left(\alpha_{d}(E) / 10^{4}\right)^{2}\left(E / E_{0}\right)^{2}\left(\lambda_{i} / R\right)^{2}$,

where the polarizability coefficient is taken from approximation (5) in units of $\left(r_{0}\right)^{3}$. For $E=E_{0}=8.3 \mathrm{~V} / \mathrm{cm}$, $R=0.1 \mathrm{~cm}, \lambda_{i}=100 r_{0}, r_{0}=10^{-4} \mathrm{~cm}$, the ratio is equal to 1.5. With decrease in the interparticle separation, the role of the dipole-dipole interaction increases.

\section{Conclusions}

It should be stressed that, in an external electric field, the elastic restoring force affects only trapped ions, which leads to the polarization of the system "dust particle - cloud of trapped ions". A simplified model of the polarization of dusty "quasiatoms" in an external electric field is presented. It is shown that the coefficient of polarizability of "a quasiatom" is very large, and the dipole-dipole forces between two dust quasiatoms can be comparable to the shadowing forces (or even larger). With increase in the electric field, the cloud of trapped ions is subjected to the field ionization. In dusty plasma, the induced dipole potential of dusty "quasiatoms" can lead to the alignment of particles and the formation of chain and multilayer structures.

The work was supported in part by Federal Targeted Program Research and Educational Staff in Innovation of Russia (contract no. 02.740.11.0109).

1. P.K. Shukla. Phys. Plasmas 8, 1791 (2001).

2. V.E. Fortov, A.G. Khrapak, S.A. Khrapak, V.I. Molotkov, and O.F. Petrov, Phys. Usp. 47, 447 (2004).

3. O. Ishihara, J. Phys. D, Appl. Phys. 40, R121 (2007).

4. S.V. Vladimirov and M. Nambu, Phys. Rev. E 52, R2172 (1995).

5. S.V. Vladimirov and O. Ishihara, Phys. Plasmas 3, 444 (1996)

6. O. Ishihara and S.V. Vladimirov, Phys. Plasmas 4, 69 (1997).

7. I.B. Bernstein and I.N. Rabinowitz, Phys. Fluids 2, 112 (1959).
8. L. Al'pert, F.V. Gurevich, A. Quarteroni, and L.P. Pitaevsky, Space Physics with Artificial Satellites (Consultants Bureau, New York, 1965).

9. J. Goree, Phys. Rev. Lett. 69, 277 (1992).

10. A.V. Zobnin, A.P.Nefedov, V.A. Sinel'shchikov, and V.E. Fortov, J. Exp. Theor. Phys. 91, 483 (2000).

11. M. Lampe, V. Gavrishchaka, G. Ganguli, and G. Joyce, Phys. Rev. Lett. 86, 5278 (2001).

12. M. Lampe, R. Goswami, Z. Sternovsky et al., Phys. Plasmas 10, 1500 (2003).

13. Z. Sternovsky, M. Lampe, and S. Robertson, IEEE Trans. Plasma Sci. 32, 632 (2004).

14. I.H. Hutchinson and L. Patacchini, Phys. Plasmas 14, 013505 (2007).

15. A.V. Zobnin, A.D. Usachev, O.F. Petrov, and V.E. Fortov, Phys. Plasmas 15, 043705 (2008).

16. G. Sukhinin, A. Fedoseev, S. Antipov, O. Petrov, and V. Fortov, Phys. Rev. E 79, 036404 (2009).

17. G.I. Sukhinin and A.V. Fedoseev, IEEE Trans. Plasma Sci. 38, 2345 (2010).

18. G. Lapenta, Physica Scripta 64, 599 (2001).

Received 22.10.10

\section{ПОЛЯРИЗАЦІЯ ПИЛОВИХ "КВАЗІАТОМІВ" У ПЛАЗМІ НИЗЬКОЇ ГУСТИНИ}

\section{Г.І. Сухінін, А.В. Федосеєв}

Р е $з$ ю м е

Наведено модель поляризації пилових частинок із захопленими іонами у зовнішньому електричному полі. Модель грунтується на самоузгодженому вирішенні інтегрального рівняння балансу для густини захоплених іонів і рівняння Пуассона для електричного потенціалу. Показано, що у слабозіштовхувальному режимі захоплені іони формують широку оболонку з максимумом, локалізованим на певній відстані, що пропорційна іонній довжині Дебая. Оцінена поляризованість пилових "квазіатомів" дуже висока і залежить від напруження зовнішнього електричного поля завдяки польовій іонізації захоплених іонів. При зростанні електричного поля пиловий квазіатом втрачає свої іони на віддалених орбітах. Радіус іонної оболонки та коефіцієнт поляризованості зменшуються. 